\title{
Proposal of Liquid Membrane-FTIR Spectroscopy to Quan- tify the Oxygen Content in Soluble Fraction of Degradative Solvent-Extraction
}

\section{Ketren Watcharakorn ${ }^{1 *}$, Muangthongon Trairat', Wannapeera Janewit ${ }^{2}$, Heishun Zen ${ }^{1}$, Toshiteru Kii', Kouichi Miura' ${ }^{1}$ and Hideaki Ohgaki'}

${ }^{1}$ Institute of Advanced Energy, Kyoto University, Gokasho, Uji, Kyoto, Japan

2Joint Graduated School of Energy and Environment, King Mongkut's University of Technology Thonburi, Bangkok, Thailand

\begin{abstract}
A new technique for the structural analysis of biomass in the liquid phase processed by the degradative solvent extraction (DSE) method, called "liquid membrane-FTIR" is proposed. The technique has been applied to analyze the hydroxyl groups of the solvent-soluble of rice straws, which was extracted in 1-methylnaphthalene (1-MN) at $350^{\circ} \mathrm{C}$ with DSE method. We found the liquid membrane-FTIR has several advantages to conventional solid membrane FTIR. 1) Easier to prepare and control the volume of the analyte, 2) Fewer chance of contamination of analyte, and 3) Sharper peaks in the spectra. A quantitative analysis of hydroxyl groups (including phenolic $\left(\mathrm{C}_{\mathrm{ar}}-\mathrm{OH}\right)$ and alcoholic $\left(\mathrm{C}_{\mathrm{al}}-\mathrm{OH}\right)$ hydroxyl) in soluble have been studied to validate the usage of the liquid membrane-FTIR technique. The result of quantification by this technique, $3.70 \%$ in weight percent of soluble on dry ash free basis, is reasonable with considering the existence of other kinds of oxygen-containing groups in soluble. The repeatability of the measurements was also examined and found that the mean difference (bias) between the measuring values of oxygen contained in hydroxyl groups by liquid membrane-FTIR and the average value of repeating measurement was very close to zero, 0.0024 , in 5 measurements with the same sample.
\end{abstract}

\section{Keywords}

Degradative solvent extraction, Liquid membrane Fourier Transform Infrared Spectroscopy (FTIR), Upgrading mechanism

\section{Introduction}

Due to the increasing of energy consumption, serious problems of global warming and health damages related to fossil fuels usage, renewable energy has increased interest in replacing not only fossil fuels but also the important chemicals $[1,2]$. Biomass is available in many countries and a wide range of sources. It is more prevalently used as an energy source compared with other renewable energy sources [3]. Biomass waste has a potential to substitute fossil fuels, therefore it is attractive to use biomass waste as feedstock for energy production because of its high carbon and hydrogen content in addition to a variety of chemical compounds [4]. However, its physical properties such as low energy density and high moisture content result in high logistic costs and reduced thermal efficiency of energy conversion [5]. The development of efficient conversion technologies of biomass to be efficient is the challenge so as to compete economically with fossil fuels. As biomass contains more oxygen than fossil fuels, it must be upgraded by using

*Corresponding author: Ketren Watcharakorn, Institute of Advanced Energy, Kyoto University, Gokasho, Uji, Kyoto, Japan, E-mail: wacharakorn.ketren.38s@st.kyoto-u.ac.jp

Received: June 23, 2017: Accepted: July 28, 2017: Published: July 31, 2017

Copyright: (c) 2017 Watcharakorn K, et al. This is an open-access article distributed under the terms of the Creative Commons Attribution License, which permits unrestricted use, distribution and reproduction in any medium, provided the original author and source are credited. 
various processes such as drying, size reduction, or thermo chemical treatment of biomass.

Degradative Solvent Extraction method (DSE) is recently developed for biomass upgrading [6]. The method has been proposed to dewater and fractionate low rank coals and biomass waste at mild conditions (below 350 $\left.{ }^{\circ} \mathrm{C}\right)$. The method successfully upgrades and fractionates biomasses [6-9]. The raw material is treated in a non-polar solvent at treatment temperature under $350{ }^{\circ} \mathrm{C}$. At this temperature, the macromolecular structure of coal/ biomass is relaxed and small molecules are extracted. The biomass sample is finally separated into three fractions; one is the unextractable fraction (called residue), the extracted portions which are the soluble in the solvent at ambient temperature (called soluble), and the deposit which is the soluble in the solvent at high temperature but precipitate at room temperature. Soluble has unique properties such as high carbon content (81.0 to $83.3 \mathrm{wt} . \%$ and low oxygen content in the range of 7.3-11 wt.\%) [6]. The study by Wannapeera, et al. [6] showed that the effective treatment temperature for deoxygenation and recovery of high carbon content in the solid product is 350 ${ }^{\circ} \mathrm{C}$. Extensive studies have been conducted such as Ashida, et al. and Zhu, et al. to investigate the mechanism of the DSE method [10,11]. To identify molecular components and structure of products from the DSE method, the researchers analyzed the composition of the solid state of the initial reactant (coal, biomass) and final products [11]. The main reactions of DSE method of biomass are thermal extraction, deoxygenation, and aromatization, however, the mechanism of the DSE method has not been fully understood so far. Therefore, we have proposed a new technique to identify the extraction product without separation from solvent would be promising in a real-time and an in situ analysis of the reactions.

The knowledge of optical properties is obviously significant for the investigation of chemical structures. The relationship between the absorption spectra and the molar fraction are directly related to the structural feature of molecules. Infrared (IR) spectroscopy provides the important information of the absorption spectra either the organic or inorganic. Therefore, various methods were developed to obtain IR spectrum of coals and carbonaceous materials. Fourier Transform Infrared (FTIR) spectroscopy is the most common spectroscopic technique used to obtain IR spectrum for molecular structure determination. Numerous FTIR studies have been conducted to investigate coal structure during the 1980s [12-16] and the band assignment of various specific functional groups of coals $[15,17]$. The conventional method known as $\mathrm{KBr}$ pellet or disk method is the most common method to obtain IR spectra for coals. The method needs fine grinding of the sample to a suitable size that reaches the maximum absorption. Furthermore, the grinding for longer times and hygroscopic property of $\mathrm{KBr}$ can produce contamination causing the problem of the background absorption and scatter $[16,18,19]$. In order to reduce the sample preparation, the reflectance method of measurement was developed to obtain IR spectra of coals from the surface of the sample. The common methods are Diffuse Reflectance (DRIFT), Attenuated Total Reflectance (ATR) and Photo Acoustic (PAS) method. DRIFT was applied to determine the relation of various band intensities to coal rank [20], to determine the product of coal from pyrolysis and heat treatment $[21,22]$. DRIFT method requires highly scattered sample and thin optical path length for quantitative measurement. In addition, band distortions could be caused by sample surface and refractive index phenomena [21]. To avoid the scattering of radiation interaction with the sample and optical thickness limitation in DRIFT, ATR technique was applied to perform quantitative analysis of coal. Although ATR spectroscopy minimizes scattering effects involved, sample limitations have impacts on quantitative studies. The solid samples have to be similar sizes and homogeneous grain, as well as good contact and the appropriate incident angle between the reflection crystal cell [23]. PAS is the other technique obtaining good IR spectra in quantitative analysis of coal without sample modification. The method is particularly good for naturally high absorption and good thermal conduction of the sample [24]. In coal analysis, the factors that affect the IR spectrum are physical states of the sample, measuring conditions, and especially the lack of reproducibility has found as a profound effect [21-24]. A quantitative analysis with IR is preferable to be conducted without modification of the analyte, neither physically nor chemically.

This work focuses on the IR measurement technique to examine the soluble fraction of biomass obtained by DSE method. The proposed technique, called 'liquid membrane-FTIR', has not yet been applied to the characterization of biomass structure in the liquid state, even though the application of a liquid cell in the FTIR measurement has been commonly performed for liquid organic compounds, such as protein structural determination [25-28]. We quantitatively determined the absolute amount of oxygen content presented in absorbance spectra of solvent-soluble by using the liquid membrane-FTIR. The result was validated with the analytical value studied by Wannapeera, et al. [6].

\section{Experimental Section}

\section{Samples}

Rice straw was used as a raw sample (approximately 6 grams-wet basis) and 1-Methyl Napthalene (1-MN, of 80 $\mathrm{mL}$ ) was used as non-hydrogen donor solvent in degradative extraction. Phenol was used as a reagent of known concentration substance of Hydroxyl Groups $(\mathrm{OH})$ in 
calibration curve determination. In order to simulate hydroxyl group contained in extracted products from DSE method, phenol was treated in 1-MN.

\section{Degradative Solvent-Extraction (DSE)}

The degradative extraction was performed in a stainless steel autoclave ( $150 \mathrm{~mL}$ of volume). After sufficiently purging the autoclave with $\mathrm{He}$, the autoclave was heated up to $350^{\circ} \mathrm{C}$ at a rate of ca. $4{ }^{\circ} \mathrm{C} / \mathrm{min}$ with holding time of $60 \mathrm{~min}$. The extracted products were separated through the opening valve to the reservoir, and the residue was filtrated at the stainless filter equipped at the bottom of the autoclave. The reservoir was cooled by water to room temperature. The precipitates extracted at room temperature were filtrated using a PTFE membrane filter to separate it as the solid extracted product (called deposit) and the extracted product in the solvent called solvent-soluble.

\section{FTIR Instrumentation}

IR spectra measurement of all samples was performed with JEOL, JIR-WINSPEC50 with a spectral range from $4000-1000 \mathrm{~cm}^{-1}$. The accumulation of spectra was 64 scans with $4 \mathrm{~cm}^{-1}$ of resolution. The time acquiring for 64 scans is approximately 150 seconds. Soluble in the solid state was measured by $\mathrm{KBr}$ pellet method and solvent-soluble in the liquid state and the reagents were measured by the liquid membrane-FTIR method. The soluble samples were prepared by the procedures as follows. At first, solvent-soluble from DSE was sampled and evaporated by a rotary evaporator at around $140{ }^{\circ} \mathrm{C}$ under reduced pressure to remove $1-\mathrm{MN}$ and to recover the extract as the solid phase. Then, the sample was dried in the vacuum oven to further minimize the solvent and the other liquid product. A small sample (typically $1.25 \mathrm{mg}$ ) was weighed and ground together with $500 \mathrm{mg} \mathrm{KBr}$. Fine ground soluble and $\mathrm{KBr}$ have dried again in a vacuum oven for 10 hours before pressed into a pellet for FTIR measurement in orderto minimize the water content in the sample. In liquid membrane-FTIR, we do not need such troublesome procedures; 1 -mm thick Calcium Fluoride $\left(\mathrm{CaF}_{2}\right)$ flat windows with the transmission range from 50,000$1,025 \mathrm{~cm}^{-1}$ was used as the window material of liquid cell because of its very low water solubility. Other window materials are not suitable for precise quantitative analysis to compare to the $\mathrm{CaF}_{2}$ window owing to their water solubility (e.g., $\mathrm{KBr}, \mathrm{NaCl}$ ) or spectral dispersion (e.g., $\mathrm{BaF}_{2}, \mathrm{ZnS}$ ) [29-31]. $\mathrm{CaF}_{2}$ is a non-hygroscopic cubic crystal resulting in very small water absorption from the atmosphere. The windows were cleaned by using acetone and wiped three times before usage. The equipment for liquid membrane-FTIR composed of two demountable metal plates for holder cell windows and sample, two $\mathrm{CaF}_{2}$-windows and a $0.1 \mathrm{~mm}$-thickness lead spacer with a rectangular space of $22 \times 10 \mathrm{~mm}^{2}$ at the middle. The as- sembling procedure of liquid membrane-FTIR, slightly put the spacer on the first $\mathrm{CaF}_{2}$ window and used a clean disposable spatula placing a few drops of liquid sample into a rectangular space of a spacer. During filling, we have made sure that the sample covers the entire space without the empty cell area or the presence of air bubbles then slowly slide the second plate window over the sample-containing lead spacer. After windows and spacer positioned properly, the demountable metal plates were used to hold the cell and assembled with four machine screws by tightening screws diagonally and carefully checked the formation of air bubbles or unfilled space. In the case of leakage from the cell at the end of the data acquisition, the cell will be dismounted and correctly reassembled and the sample will need to be reloaded and remeasured. To eliminate moisture and better stability of the measurement, 2 liters per minute of nitrogen gas was continuously purging into the sample compartment of the FTIR. The agreement and reliability of technique by quantifying the repeatability of measurement were tested. Five replicated measurements of solvent-soluble were performed by liquid membrane-FTIR under the same measurement condition. Each sample was put in an ultrasonic bath to prepare the homogeneity before measurement.

\section{Calibration}

The main process of DSE method is the deoxygenating $[10,11]$, the oxygen containing in solvent-soluble was examined. In order to quantify the oxygen in solvent-soluble, phenol reagent of various concentrations in 1-MN was used for establishing the calibration curve. The data from the spectral region associated with phenolic groups, namely, the oxygen-hydrogen stretching regionwas presented in this study. Based on the study of Painter, et al. and Miura K, et al. [32,33], there are hydrogen bonds formed by hydroxyl groups, acid/base complex base, and carboxylic acid dimmers. The band assignment and chemical structure of $\mathrm{OH}$ stretching bands were illustrated in Table 1. Background spectra of air were collected for every sample immediately before the collection of the sample single-beam spectrum. The IR spectra of all samplewere subtracted with 1-MN spectrum and the linear baseline correction. The spectrum of $\mathrm{OH}$-stretching

Table 1: Band assignments of sub-peak and variation range of positions and widths of solvent-soluble [32,33].

\begin{tabular}{|l|l|l|}
\hline $\begin{array}{l}\text { Band assignments of hydrogen } \\
\text { bonds }\end{array}$ & $\begin{array}{l}\text { Band position } \\
\left(\mathrm{cm}^{-1}\right)\end{array}$ & $\begin{array}{l}\text { Width } \\
\left(\mathrm{cm}^{-1}\right)\end{array}$ \\
\hline Free OH groups & 3611 & 30 \\
\hline OH-m hydrogen bonds & $3530 \pm 10$ & $50 \pm 20$ \\
\hline Self-associated $n$-mers $(\mathrm{n}>3)$ & 3400 & $100 \pm 10$ \\
\hline OH-ether O hydrogen bonds & $3280 \pm 35$ & $70 \pm 15$ \\
\hline Tightly bound cyclic OH tetramers & $3150 \pm 50$ & $60 \pm 10$ \\
\hline OH-N (acid/base structure) & $3100-2800$ & - \\
\hline COOH dimers & 2640 & - \\
\hline
\end{tabular}


vibration relates to hydrogen bonding appears between $3650-3100 \mathrm{~cm}^{-1}$ and $3650-3400 \mathrm{~cm}^{-1}$ of solvent soluble and phenol, respectively, therefore the straight baseline was drawn between these wave numbers by setting the $y$-intercept and slope as a baseline correction. The baseline was drawn from the inflection point around 3640 $\mathrm{cm}^{-1}$ to the absorbance minima between 3050 and 3000 $\mathrm{cm}^{-1}$ for solvent soluble and between 3390 and $3350 \mathrm{~cm}^{-1}$ for the reagent phenol. The number, the sub-peak position, the width and their variation ranges were assigned in Table $1[32,33]$. Gaussian distribution was adopted as a peak shape which was considered as effective enough for each absorption band by Miura and co-workers [34-36]. All parameters were used as the initial guesses of each sample. Which used a set of preselected peaks, whose position and width were held and whose amplitude is varied to reproduce the experimental spectrum. A spectrum was processed by multi-peak fit function by software IGOR Pro 6.31. This analysis function uses the Levenberg-Marquardt algorithm, which is a form of least squares fitting. The fit is finished when the rate at Chi-square decreases is small enough. The peaks were derived from the fitted spectrum and the peak areas were obtained from this function analysis.

In absorption spectroscopy, the absorbance obtained from the measurement is the relationship as known as the Beer-Lambert law;

$$
I=I_{0}^{*} 10^{-\left(\frac{\alpha}{L C}\right)} \quad\left(\mathrm{W} . \mathrm{cm}^{-2}\right)
$$

Where $I$ and $I_{0}$ are the intensity of light passing though the absorbing sample and incident on the sample, respectively, $\alpha$ is the absorptivity or the absorption coefficient, $L$ is the path-length of absorption and $c$ is the concentration of the sample. The measured value used of $I$ and $I_{0}$ to compute the absorbance, $A$, defined as

$$
A=\log \left(I / I_{0}\right)=\alpha L c
$$

The equation states a linear relationship between the absorbance and the concentration of the absorbing species. The plot between the measured absorbance (y-axis) and concentrations ( $\mathrm{x}$-axis) is called a calibration curve. The resulting slope is the absorptivity dividing by the path-length. If the absorbance is obtained by measuring from the known concentration compound at a certain path-length, the absorptivity of the compound can calculate.

It is known that several researchers attempted to estimate hydrogen bonds in coal by various methods. In order to quantify the amount of oxygen in $\mathrm{OH}$ stretching region (3650-3100 $\mathrm{cm}^{-1}$ ) of our sample spectrum, the study has adopted the method developed by Miura and co-workers [34-36] to investigate the distribution of hydrogen bonds in the $\mathrm{OH}$-stretching band of coal by analyzing FTIR spectra. The According to Beer-Lambert law, the analytical equation can be written as

$$
A_{i}=\alpha_{i} n_{O H, i},
$$

Where $A_{i}$ is the integral intensity or the sub-peak area of the hydrogen bond $i$ from the curve fitting result, $\alpha_{i}$ is the absorptivity of the $i^{\text {th }}$ peak and $n_{\mathrm{OH}, i}$ is the estimated amount of $\mathrm{OH}$ for the $i^{\text {th }}$ peak. Therefore the estimated amount of $\mathrm{OH}$ for the $i^{\text {th }}$ peak is

$$
n_{O H, i}=\frac{A_{i}}{\alpha_{i}} \quad \quad\left(\mathrm{mg} \cdot \mathrm{ml}^{-1}\right)
$$

Regarding consist of absorption bands in $\mathrm{OH}$ stretching region as Table $1 ; 6 \mathrm{OH}$ stretch bands. The absorptivity of each band, the $i^{\text {th }}$, must be determined to convert the individual intensities to corresponding amounts of $\mathrm{OH}$ groups. The studies of Miura and co-workers [34-36] arbitrarily assumed a similar relationship for the change in absorption intensity of hydrogen-bonded $\mathrm{OH}$, and estimated the proportional constant from the intensity and the $\Delta v_{\mathrm{OH}}$ values on the basis of the work of Detoni and co-workers [37]. The absorptivity of hydrogen bands is as

$$
\alpha_{O H}=\alpha_{O H, O}\left(1+0.0147 \Delta v_{O H}\right)\left(\mathrm{ml} \mathrm{mg}^{-1} \cdot \mathrm{cm}^{-1}\right)
$$

Where $\alpha_{O H}$ is the absorptivity of hydrogen-bonded $\mathrm{OH}, \alpha_{\mathrm{OH}, \mathrm{O}}$ is the absorptivity of the free $\mathrm{OH}$ and $\Delta v_{\mathrm{OH}}$ is the wave number shift to hydroxyl groups in hydrogen bonds relative to the position of free $\mathrm{OH}$ groups (3611 $\mathrm{cm}^{-1}$ ). Thus the amounts of $\mathrm{OH}$ contributing to the different hydrogen bonds can be calculated if $\alpha_{\text {ОН.О }}$ can estimate [36]. Therefore the absorptivity of each hydrogen bond was assumed to be represented by Equation (5) in this analysis is as

$$
\alpha_{i}=\alpha_{O H, O}\left(1+0.0147\left(\Delta v_{O H, i}\right)\right) \quad\left(\mathrm{ml} \mathrm{mg}^{-1} \cdot \mathrm{cm}^{-1}\right)
$$

Then the amount of the $\mathrm{OH}$ corresponding to the $i^{\text {th }}$ peak, $n_{\mathrm{OH}, i}$ is given by

$$
n_{O H, i}=A_{i} /\left\{\alpha_{O H, O}\left(1+0.0147\left(\Delta v_{O H, i}\right)\right)\right\} . \quad\left(\mathrm{mg} \cdot \mathrm{ml}^{-1}\right)
$$

The total amount of $\mathrm{OH},\left(n_{\mathrm{OH}, \mathrm{Total}}\right)$, related to the intensity of each absorption band can be calculated by

$$
n_{O H, \text { Total }}=\Sigma_{i} n_{O H, i} . \quad\left(\mathrm{mg} \cdot \mathrm{ml}^{-1}\right)
$$

In order to determine the total amount of $\mathrm{OH}$ of solvent-soluble, the absorptivity of free $\mathrm{OH}, A, a$, is needed to estimate. Due to $A, a$ is the slope between the absorbance and the concentration of free $\mathrm{OH}$, the calibration curve of free $\mathrm{OH}$ is established. In our study used Phenol treated in 1-MN was used as a reagent of known concentration substance of Hydroxyl Groups $(\mathrm{OH})$. By applying curve fitting method and peak resolving the spectra of the reagent at different concentration, the calibration curve 

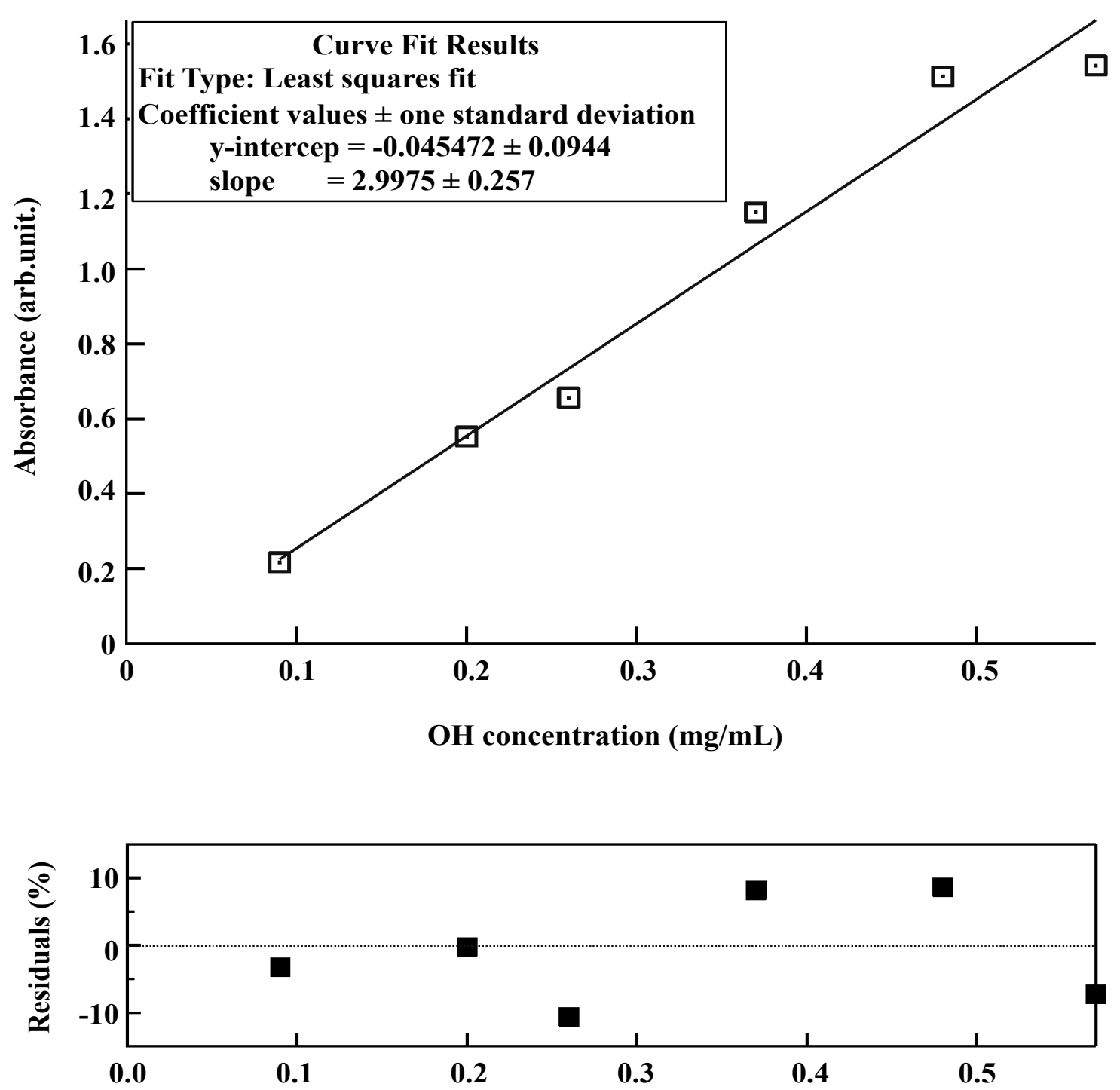

Figure 1: Calibration curve of the free $\mathrm{OH}$ of phenol treated in the 1-MN solvent and the percent residuals of least squares fitting.

of free $\mathrm{OH}$ can be established. The absorptivity of free $\mathrm{OH}, A, a$, is determined from the value of the slope at a certain path-length of the plot between the absorbance of free $\mathrm{OH}$ (y-axis) and the concentration of free $\mathrm{OH}$ ( $\mathrm{x}$-axis) presented as Figure 1. Least squares curve fitting techniques and the residual standard deviation had been used to improve the calibration curve of free $\mathrm{OH}$.

\section{Results and Discussion}

IR spectra of the liquid sample were obtained from the liquid membrane-FTIR method and the conventional $\mathrm{KBr}$ pellet method for the solid sample. Figure 2 shows the FTIR spectra of solvent-soluble and soluble in solid phase performed at $350^{\circ} \mathrm{C}$ of treatment temperature. They were measured at room temperature. The measurements provided the $\mathrm{OH}$ region $\left(3750-2400 \mathrm{~cm}^{-1}\right)$ and aromatic-aliphatic region $\left(3200-1360 \mathrm{~cm}^{-1}\right)$. It is clear that the spectrum obtained by liquid membrane-FTIR provides more structural information, as the peaks of the liq- uid membrane-FTIR are sharper and more distinct than those obtained by the conventional $\mathrm{KBr}$ pellet method. Regarding the appearing of sudden peaks obtained from liquid membrane-FTIR spectrum (upper-spectra) results from 1-MN spectrum, and the slight mismatch between the background spectrum and the sample spectrum. We thought these sudden peaks effect is one of the drawback of liquid-membrane FTIR in measuring of the sample within the solvent.

Figure 3 shows the results of peak deconvolution by curve-fitting and the hydrogen bonds of solvent-soluble and phenol in 1-MN as an example. The peak of hydrogen bonds in FTIR spectrum of the solvent-soluble and the reagent phenol appeared in the region of 3650-3100 $\mathrm{cm}^{-1}$ and $3650-3400 \mathrm{~cm}^{-1}$, respectively. The peak positions were slightly changed, depending on the sample by referring to curve fitting of each spectrum. The four types of hydrogen bonds in solvent-soluble were found as $\mathrm{OH}-\pi$ at $3540 \mathrm{~cm}^{-1}, \mathrm{OH}-\mathrm{OH}$ at $3400 \mathrm{~cm}^{-1}$, OH-ether 


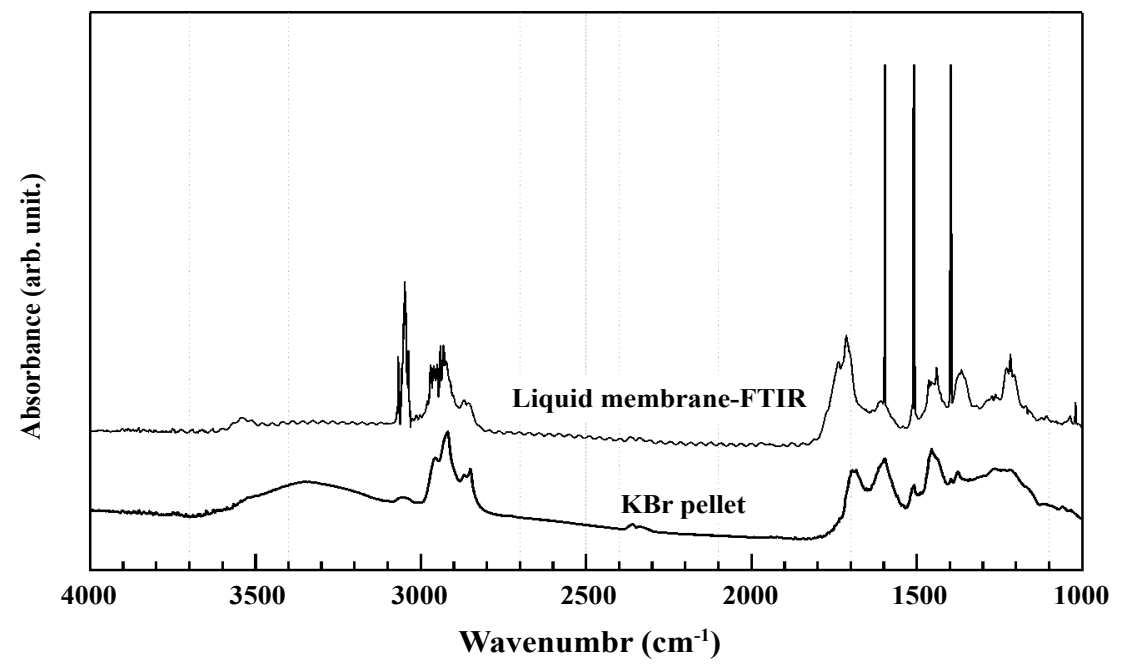

Figure 2: The typical FTIR spectra of soluble obtained from liquid membrane-FTIR and KBr pellet.
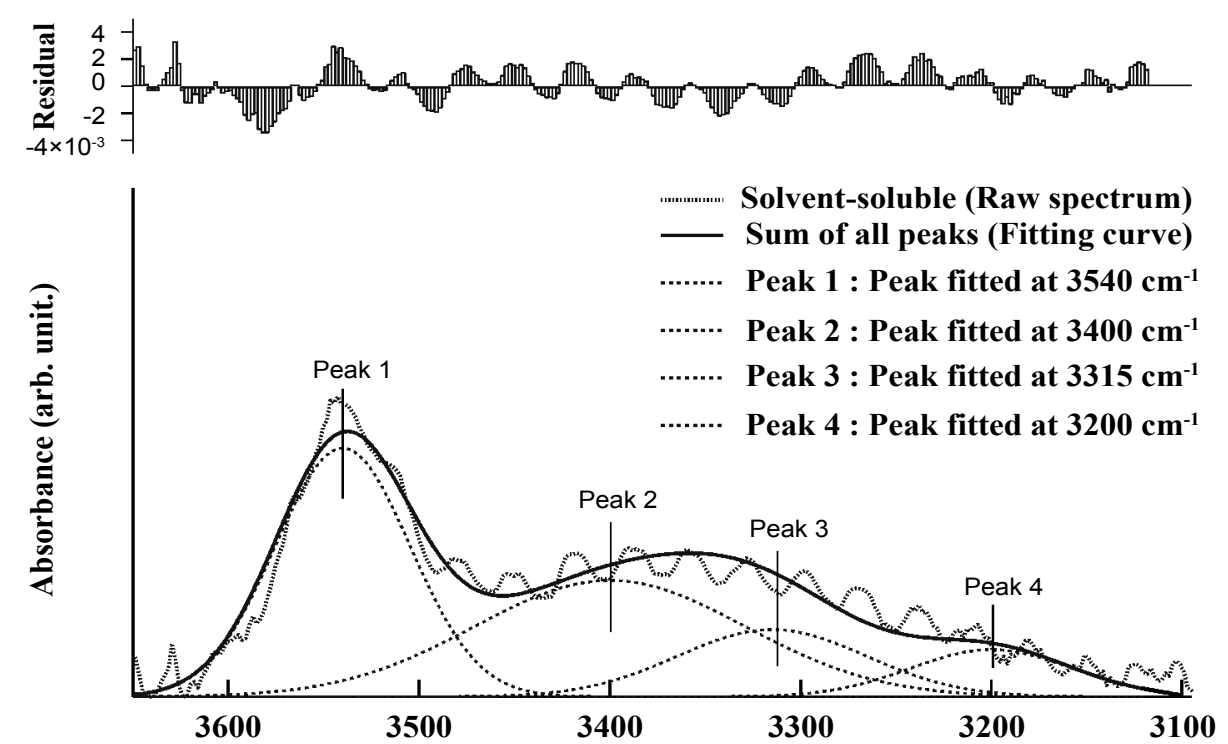

Wavenumber $\left(\mathrm{cm}^{-1}\right)$

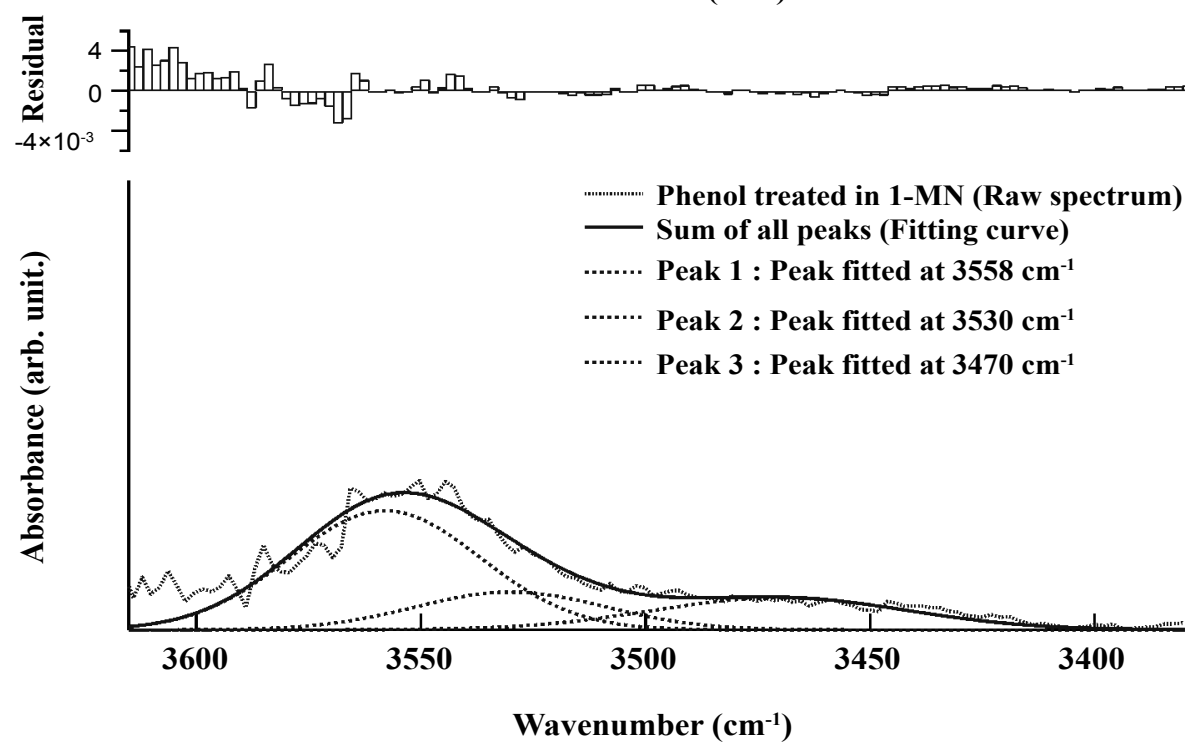

Figure 3: FTIR spectrum obtained from liquid membrane-FTIR (above) FTIR spectrum of solvent-soluble performed at 350 ${ }^{\circ} \mathrm{C}$ treatment temperature (bottom) FTIR spectrum of $0.2 \mathrm{mg} / \mathrm{mL}$ of phenol in 1-MN. 
Table 2: Descriptive of reliability [38-40].

Statistic indices

\begin{tabular}{|l|l|l|l|l|l|}
\hline Number of replicated & Mean & Varian & Standard deviation & Standard error of the mean & $\begin{array}{l}\boldsymbol{p} \text {-value } \\
\text { (Shapiro-Wilk) }\end{array}$ \\
\hline 5 & 3.70 & 0.03 & 0.17 & 0.07 & 0.12 \\
\hline
\end{tabular}

Relative and absolute reliability indices

\begin{tabular}{|l|l|l|l|l|l|}
\hline Mean diff (Bias) & $\begin{array}{l}\text { SD diff } \\
\text { (between subject) }\end{array}$ & $\begin{array}{l}\mathbf{9 5 \%} \text { LOA } \\
(\mathbf{9 5 \%} \mathrm{CI}) \text { LB }\end{array}$ & $\begin{array}{l}\mathbf{9 5 \%} \text { LOA } \\
(\mathbf{9 5 \%} \mathrm{CI}) \text { UB }\end{array}$ & Within- subject variance & CR \\
\hline 0.0024 & 0.16 & -0.31 & 0.32 & 0.03 & \pm 0.44 \\
\hline
\end{tabular}

Mean diff is the mean of the difference between the measured values and the average value; SD diff is the standard deviation of the different value; 95\% LOA = Mean diff $\pm 1.96 \times$ SD diff; 95\% LOA LB (95\% Cl of the LOA) $=95 \%$ Limits of agreement Lower Boundary (95\% Confidence intervals of the limits of agreement); 95\% LOA UB (95\% Cl of the LOA) $=95 \%$ Limits of agreement Upper Boundary (95\% Confidence intervals of the limits of agreement); CR (Repeatability Coefficient) $=\mathrm{SD}$ diff $\times 1.96 \sqrt{2}=$ the smallest real different.

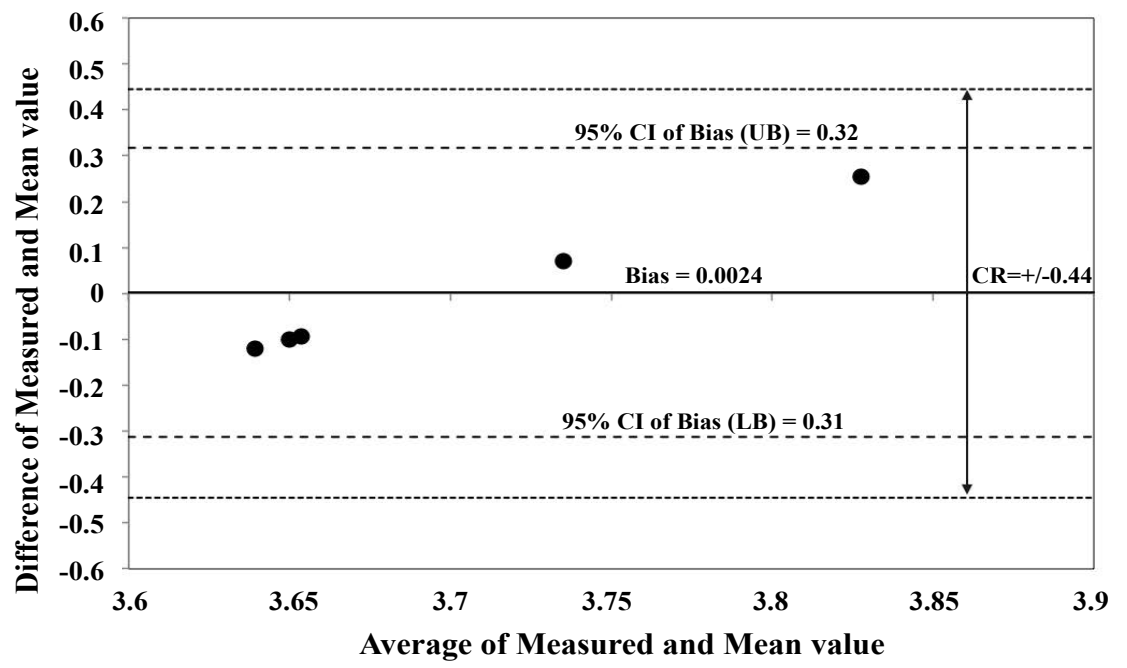

Figure 4: Differences between the measuring values of oxygen contained in hydroxyl by liquid membrane-FTIR and the average values of repeating measurement against their means. The solid line represents the mean different; upper and lower dashed line shows the mean different $\pm 1.96 \mathrm{SD}$, each with $95 \% \mathrm{Cl}$, and the upper and the lower dotted lines show the Repeatability Coefficient (CR) of the technique.

at $3315 \mathrm{~cm}^{-1}$ and cyclic $\mathrm{OH}-\mathrm{OH}$ at $3200 \mathrm{~cm}^{-1}$. Free $\mathrm{OH}$ at $3558 \mathrm{~cm}^{-1}$, OH dimer at $3530 \mathrm{~cm}^{-1}$ and $\mathrm{OH}$ trimer at 3470 $\mathrm{cm}^{-1}$ were resolved from phenol reagent in $1-\mathrm{MN}$ solvent. By curve resolving, the calibration curve of the free $\mathrm{OH}$ can be obtained.

Figure 1 shows the calibration curve of the free $\mathrm{OH}$ of phenol in the 1-MN solvent and the percent residuals of least squares fitting. The regression coefficient of the fitting line of peak area to $\mathrm{OH}$ concentration represented the free $\mathrm{OH}$ absorptivity. As the absorptivity of free $\mathrm{OH}$, $\alpha_{\mathrm{OH}, \mathrm{O}}$ is obtained and the sub-peak area of the hydrogen bond of solvent-soluble in $\mathrm{OH}$ region results from the curve fitting, by applying Equation (8) the total amount of $\mathrm{OH}$ in solvent-soluble can be obtained. By excluding the estimated value of the moisture and ash content and the oxygen containing in $\mathrm{OH}$ in solvent-soluble is determined, the oxygen content in weight percent of soluble on dry ash free basis is obtained.

Calculated from the spectra and the calibration curve, the repeatability of the method was accessed by five times measurements. The results in weight percent of soluble on dry ash free basis were 3.60, 3.77, 3.58, 3.96 and 3.61. The estimated amount of oxygen obtained from hydroxyl groups in solvent-soluble with the precision was 3.70 $\pm 0.17 \%$. Considering the existence of other oxygen-containing functionalities, the oxygen from hydroxyl group in the solvent soluble should be less than $7.3-11 \%$ which is deduced from literature [6]. The distribution of oxygen-containing functional group in carbonaceous material is not only comprised of Phenolic $\left(\mathrm{C}_{\mathrm{ar}}-\mathrm{OH}\right)$ and Alcoholic $\left(\mathrm{C}_{\mathrm{al}} \mathrm{OH}\right)$ Hydroxyl Groups, but also of Carboxyl Groups (R-COOH), Carbonyl Groups (R-CO), Ethers (R-O-R') and Quinone Groups $\left(\mathrm{C}_{\mathrm{ar}}=\mathrm{O}\right)$ [41]. The reproducibility of measurements, the agreement between different measurements in the same sample can be represented as reliability indices in Table 2 . In order to test the repeatability of the method, the measurements remain consistent over repeated tests of the same sample under identical conditions is considered in the study. 
An experiment is reliable if it yields consistent results of the same measure. We used a plot of a Bland Altman as shown in Figure 4 in order to see their difference between the repeated measurement value by this method. If there is agreement and the result in a $95 \%$ prediction interval, the differences are assumed normally distributed. The normally distribute of data used Shapiro-Wilk test which can perform the number of sample from 3 to 50 elements. We arrived at 0.12 of p-value interpolated from Shapiro-Wilk test. Since p-value is more than 0.05 , we retained the null hypothesis that the differences are normally distributed. The mean difference between the measuring values of oxygen contained in hydroxyl groups by liquid membrane-FTIR and the average value of repeating measurement (bias) was very close to zero, 0.0024. It indicated that this average discrepancy between the measurement values with the mean value was very small. The estimated limits of agreement range within expected $95 \%$ of the confidence interval for the bias was $[-0.31,0.32]$. The smallest possible change of the bias or the Repeatability Coefficient (CR) was \pm 0.44 . The diagram as plotted in Figure 4 illustrated the repeatability of the method by comparing repeated measurements by liquid membrane-FTIR within the same sample. The average difference points were found to be close to the bias (baseline), within the $95 \%$ confidence interval.

\section{Conclusions}

We proposed the liquid membrane-FTIR technique which is suitable for the quantitative analysis of the soluble fraction from DSE method. The measurement can be performed with soluble without removing solvent that can avoid the contaminations during the sample preparation.

The results of the quantitative analysis of the oxygen content of the hydroxyl groups in a solvent-soluble sample extracted from biomass by DSE, 3.7\%, was a reasonable one in comparison with the previous result. The reproducibility of this method was shown to be excellent. It is worth to note that this technique could be applied to real-time and in-situ analysis for DSE or other solvent processes.

The deduced quantity of the oxygen by this technique still needs to be checked by other techniques, such as $\mathrm{H}-\mathrm{NMR}$, because the solvent-soluble sample comprises several functional oxygen groups whose weight percentages are not well known. Even though, our proposed technique can provide a reliable quantity of hydroxyl groups in biomass, which will help to deepen our understanding of the structure of biomass and reaction mechanism of DSE.

\section{References}

1. (2013) BP Statistical review of world energy June 2013.

2. EIA (2011) Annual Energy Review 2011. U.S. Energy Information Administration. Washington, DC.
3. IPCC (2012) Renewable energy sources and climate change mitigation. Special Report of the Intergovernmental Panel on Climate Change. Cambridge, Cambridge University Press.

4. Lv D, Xu M, Liu X, Zhan Z, Li Z, et al. (2010) Effect of cellulose, lignin, alkali and alkaline earth metallic species on biomass pyrolysis and gasification. Fuel Process Technol 91: 903-909.

5. Liu Y, Aziz M, Fushimi C, Kansha Y, Mochidzuki K, et al. (2012) Exergy analysis of biomass drying based on selfheat recuperation technology and its application to industry : a simulation and experimental study. Industrial and Engineering Chemistry Research 51: 9997-10007.

6. Wannapeera J, Li X, Worasuwannarak N, Ashida R, Miura $K$ (2012) Production of high-grade carbonaceous materials and fuel having similar chemical and physical properties from various types of biomass by degradative solvent extraction. Energy Fuels 26: 4521-4531.

7. Li X, Ashida R, Miura K (2012) Preparation of high-grade carbonaceous materials having similar chemical and physical properties from various low-rank coals by degradative solvent extraction. Energy Fuels 26: 6897-6904.

8. Li X, Zhu X, Xiao L, Ashida R, Miura K, et al. (2014) Degradative solvent extraction of demineralized and ion-exchanged low-rank coals. J Fuel Chem Technol 42: 897-904.

9. Zhu X, Zhang Z, Zhou Q, Cai T, Qiao E, et al. (2015) Upgrading and multistage separation of rice straw by degradative solvent extraction. J Fuel Chem Technol 43: 422-428.

10. Ashida R, Takahashi R, Kawase M, Miura K (2015) Upgrading mechanism in degradative solvent extraction of biomass wastes.

11. Zhu X, Xue Y, Li X, Zhang Z, Sun W, et al. (2016) Mechanism study of degradative solvent extraction of biomass. Fuel 165: 10-18.

12. Painter PC, Snyder RW, Starsinic M, Coleman MM, Kuehn DW, et al. (1981) Concerning the Application of FT-IR to the Study of Coal: A Critical Assessment of Band Assignments and the Application of Spectral Analysis Programs. Applied Spectroscopy 35: 475-485.

13. Painter PC, Starsinic M, Squires E, Davis A (1983) Concerning the $1600 \mathrm{~cm}^{-1}$ region in the i.r. spectrum of coal. Fuel 62: 742-744.

14. Solomon PR (1981) Relation between coal aromatic carbon concentration and proximate analysis fixed carbon. Fuel 60: 3-6.

15. Solomon PR, Carangelo RM (1988) FT-ir analysis of coal: 2. Aliphatic and aromatic hydrogen concentration. Fuel 67 : 949-959.

16. Sobkowiak M, Painter PA (1995) A comparison of drift and $\mathrm{KBr}$ pellet methodologies for the quantitative analysis of functional groups in coal by infrared spectroscopy. Energy \& Fuels 9: 359-363.

17. Solomon PR, Carangelo RM (1982) FTIR analysis of coal. 1. Techniques and determination of hydroxyl concentrations. Fuel 61.

18. Friedel RA, Retcofsky HL, Queiser JA (1967) Advance in coal spectrometry, absorption spectrometry. U.S. Department of the Interior, Bureau of Mines, Bulletin 640. 
19. John C (2006) Interpretation of Infrared Spectra, A Practical Approach. Encyclopedia of Analytical Chemistry.

20. Fuller MR, Griffiths PR (1978) Diffuse reflectance measurements by infrared Fourier transform spectrometry. Anal Chem 50: 1906-1910.

21. Sobkowiak M and Painter PA (1995) A comparison of drift and $\mathrm{KBr}$ pellet methodologies for the quantitative analysis of functional groups in coal by infrared spectroscopy. Energy Fuels 9: 359-363.

22. Glover G, van der Walt TJ, Glasser D, Prinsloo NM, Hildebrandt D (1995) DRIFT spectroscopy and optical reflectance of heat-treated coal from a quenched gasifier. Fuel 74: 1216-1219.

23. Thomasson J, Coin C, Kahraman H, Fredericks PM (2000) Attenuated total reflectance infrared microspectroscopy of coal. Fuel 79: 685-691.

24. Jorge A Orrego-Ruiz, Rafael C, Enrique Mejia-O (2011) Study of Colombian coals using photoacoustic Fourier transform infrared spectroscopy. Int J of Coal Geology 85: 307-310.

25. Dong A, Huang P, Caughey WS (1990) Protein secondary structures in water from second-derivative amide I infrared spectra. Biochemistry 29: 3303-3308.

26. Yu S, Wuu A, Basu R, Holbrook MR, Barrett AD, et al (2004) Solution structure and structural dynamics of envelope protein domain III of mosquito-and tick-borne flaviviruses. Biochemistry 43: 9168-9176.

27. Yu S, Mei FC, Lee JC, Cheng X (2004) Probing cAMP-dependent protein kinase holoenzyme complexes I alpha and II beta by FT-IR and chemical protein footprinting. Biochemistry 43: 1908-1920.

28. Huayan Y, Shouning Y, Jilie K, Aichun D, Shaoning Y (2015) Obtaining information about protein secondary structures in aqueous solution using Fourier transform IR spectroscopy. Nature Protocols 10: 382-396.

29. Application Note-0602, Spectroscopic creativity, PIKE technologies.
30. Katsumi H, FTIR and water, FTIR Talk Letter Vol.19, Shimadzu.

31. Nasse MJ, Ratti S, Giordano M, Hirschmugl CJ (2009) Demountable liquid/flow cell for in vivo infrared microspectroscopy of biological specimens. Appl Spectrosc 63: 11811186.

32. Painter PC, Sobkowiak M, Youtcheff J (1987) FT-i.r. study of hydrogen bonding in coal. Fuel 66: 973-978.

33. Miura K, Mae K, Sakurada K (1992) Flash pyrolysis of coal following thermal pretreatment at low temperature. Energy Fuels 6: 16-21.

34. Miura K, Kazuhiro M, Li W, Takumi K, Akido K (1999) In Situ FTIR Measurement of the change in hydrogen bonding of coal through heat treatment. ASC Fuel Div 44: 642-646.

35. Miura K, Kazuhiro M, Fumi-aki M (1997) A new method to estimate hydrogen bonds in coal by utilizing ftir and dsc. Am Chem Soc Fuel Div 42: 209-213.

36. Miura K, Mae K, Li W, Kusakawa T, Morozumi F, et al. (2001) Estimation of hydrogen bond distribution in coal through the analysis of $\mathrm{OH}$ stretching bands in Diffuse Reflectance Infrared Spectrum Measured by in-situ technique. Energy Fuels 15: 599-610.

37. Detoni S, Hadzi D, Smerkolj R (1970) Hydrogen bonding in some adducts of oxygen bases with acids. Part VI. Infrared and dielectric studies of adducts with isothiocyanic acid. $\mathrm{J}$ Chem Soc A 2851-2859.

38. De Vhcw, Terwee CB, Knol DL, Bouter LM (2006) When to use agreement versus reliability measures. J Clin Epidemiol 59: 1033-1039.

39. National Institute for Standards and Technology (2007) Guidelines for Evaluating and Expressing the Uncertainty of NIST Measurement Results.

40. Bartlet JW, Frost C (2008) Reliability, repeatability and reproducibility: analysis of measurement errors in continuous variables. Ultrasound Obstet Gynecol 31: 466-475.

41. Krevelen DWV (1993) Coal, typology-physics-chemistry-constitution. ( $3^{\text {rd }}$ edn), Elsevier Science Publishers B.V. 